\title{
Mathematics of Random Growing Interfaces
}

\author{
Mathew D. Penrose and J. E. Yukich ${ }^{1}$ \\ University of Durham and Lehigh University
}

\begin{abstract}
We establish a thermodynamic limit and Gaussian fluctuations for the height and surface width of the random interface formed by the deposition of particles on surfaces. The results hold for the standard ballistic deposition model as well as the surface relaxation model in the off-lattice setting. The results are proved with the aid of general limit theorems for stabilizing functionals of marked Poisson point processes.
\end{abstract}

\section{Introduction}

Aggregation and growth processes associated with the deposition of particles on surfaces is of considerable scientific and practical interest (Barabási and H. E. Stanley [1], Cumberland and Crawford [2], Vicsek [8]). Of special interest is the morphology of the random interface separating the growing material from its environment. The surface morphology, which involves non-equilibrium growth behavior, exhibits scaling and is thought to evolve to a steady state without a characteristic time or length scale (Family [4], Family and Vicsek [5]).

This note rigorously develops the mathematical limit theory of the random particle deposition model in which random size $(d+1)$-dimensional balls ('particles') rain down sequentially at random onto a $d$ dimensional substrate $Q_{n}$ of volume $n^{d}$. The substrate $Q_{n}$ is assumed initially flat but it need not be oriented perpendicularly to

Department of Mathematical Sciences, University of Durham, South Road, Durham DH1 3LE, England: mathew.penrose@durham.ac.uk

Department of Mathematics, Lehigh University, Bethlehem PA 18015, USA: joseph.yukich@lehigh.edu

${ }^{1}$ Research supported in part by NSA grant MDA904-01-1-0029 
the particle trajectories. Assume that particle sizes are independent and identically distributed with diameter bounded by a constant $D_{1}$.

When a particle arrives on the existing agglomeration of deposited balls, it may stick to the first particle it contacts, which may result in lateral growth and 'overhangs' (the ballistic deposition (BD) model). Alternatively, the particle may slip and roll over existing particles, undergoing vertical and horizontal displacements, stopping when it reaches a position of lower height (the surface relaxation (SR) model). In this model, the particle diffuses along the interface up to a finite distance, say $D_{2}$ (c.f. $[4,1]$ ). Also, only those existing particles within distance $D_{1}+D_{2}$ of the new particle affect its motion. The finite displacement assumption is consistent with the presence of moderately sticky particles.

Both the BD and SR models have been intensely studied in the lattice and offlattice (continuum) settings (see [1] for an overview of the enormous literature). We focus here on the continuum setting.

Let $\mathcal{X} \subset \mathbb{R}^{d} \times[0, \tau]$ denote a finite point set of particles, which are released from 'infinity' onto the substrate $\mathbb{R}^{d}$. Represent points in $\mathcal{X}$ by $(X, t)$, where $X \in \mathbb{R}^{d}$ denotes the spatial location (center) of the incoming particle and $t$ its time of arrival. The parameter $\tau$ is the 'mean deposition intensity', i.e., the average number of particles arriving per unit volume in $\mathbb{R}^{d}$.

The 'active zone' or the 'interface' of the resulting agglomeration formed through either the BD or SR process is defined as follows. A particle is termed 'exposed' if there is a positive chance that a subsequent incoming particle could strike it directly. The active zone is the set $A(\mathcal{X})$ of exposed particles belonging to the agglomeration induced by $\mathcal{X}$.

Given $\mathcal{X}:=\left(X_{1}, \ldots, X_{k}\right) \subset \mathbb{R}^{d} \times[0, \tau]$, let $X_{i}^{\prime}, 1 \leq i \leq k$, denote the position of $X_{i}$ after displacement. Let $h\left(X_{i}^{\prime}\right):=h\left(\mathcal{X}, X_{i}^{\prime}\right)$ denote the height of $X_{i}^{\prime}$ above the substrate $\mathbb{R}^{d}$. Since the maximum diameter is $D_{1}$ and the maximum displacement is $D_{2}$, the height $h(X)$ of a particle $(X, t)$ is a random variable determined by points in the existing configuration within a distance $D_{1}+D_{2}$ of $X$.

Define the height functional

$$
H(\mathcal{X}):=\sum_{X_{i}^{\prime} \in A(\mathcal{X})} h\left(X_{i}^{\prime}\right), \quad \mathcal{X} \subset \mathbb{R}^{d} \times[0, \tau]
$$

and the mean height functional

$$
\bar{H}(\mathcal{X}):=\frac{H(\mathcal{X})}{|A(\mathcal{X})|}, \quad \mathcal{X} \subset \mathbb{R}^{d} \times[0, \tau] .
$$

The squared width of $X_{i} \in \mathcal{X}$ about the mean is

$$
w\left(X_{i}^{\prime}\right):=w\left(\mathcal{X}, X_{i}^{\prime}\right):=\left(h\left(X_{i}^{\prime}\right)-\bar{H}(\mathcal{X})\right)^{2}, \quad \mathcal{X} \subset \mathbb{R}^{d} \times[0, \tau] .
$$


The width functional is

$$
W(\mathcal{X}):=\sum_{X_{i}^{\prime} \in A(\mathcal{X})} w\left(X_{i}^{\prime}\right), \quad \mathcal{X} \subset \mathbb{R}^{d} \times[0, \tau]
$$

and the surface width functional is

$$
\bar{W}:=\bar{W}(\mathcal{X}):=\left(\frac{W(\mathcal{X})}{|A(\mathcal{X})|}\right)^{1 / 2}, \quad \mathcal{X} \subset \mathbb{R}^{d} \times[0, \tau],
$$

i.e., $\bar{W}$ is the root mean square of fluctuations of heights of particles in the active zone.

We are interested in the mean height and surface width functionals when $\mathcal{X}$ is the realization of a rate one homogeneous space time Poisson point process $\mathcal{P}_{n, \tau}$ on $Q_{n} \times[0, \tau], Q_{n} \subset \mathbb{R}^{d}$. In this case we write $A(n, \tau):=A\left(\mathcal{P}_{n, \tau}\right)$ for the active zone, and do not distinguish between the active zones for the BD and SR models. Let $\left\{P_{i}\right\}$ denote the points in $\mathcal{P}_{n, \tau}$.

Define the random height functional $H(n, \tau):=H\left(\mathcal{P}_{n, \tau}\right)$ and the mean random height functional $\bar{H}(n, \tau):=\bar{H}\left(\mathcal{P}_{n, \tau}\right)$. Similarly, define the random width functional $W(n, \tau):=W\left(\mathcal{P}_{n, \tau}\right)$ and the random surface width functional $\bar{W}(n, \tau):=\bar{W}\left(\mathcal{P}_{n, \tau}\right)$.

Considerable research has focused on the qualitative properties of the random surface width $\bar{W}(n, \tau)$. Its analysis is rendered difficult by the presence of spatially correlated squared heights $\left(h\left(P_{i}^{\prime}\right)-\bar{H}\right)^{2}, P_{i}^{\prime} \in A(n, \tau)$. Both the random height and random width functionals involve triangular arrays of identically distributed but spatially correlated summands.

Beginning with the seminal papers [4], [5], and Kardar, Parisi, and Zhang [6], it is widely held that the surface width exhibits both space and time scaling and is governed by the dynamic scaling relation

$$
\bar{W}(n, \tau) \approx n^{\alpha} f\left(\tau / n^{\alpha / \beta}\right)
$$

where the scaling function $f$ satisfies

$$
f(x) \approx x^{\beta}, \quad x \ll 1,
$$

and $f(x) \approx C$ for $x \gg 1$. Here, $\alpha$ is called the roughness exponent [1] and $\beta$ is the growth exponent; $\alpha$ describes the dependence of the surface width in the long time limit $\tau \rightarrow \infty$ on the linear size $n$, whereas $\beta$ reveals the dependence on time $\tau$. In the region $\tau \ll n$, we have

$$
\bar{W}(n, \tau) \approx \tau^{\beta}
$$


whereas for $\tau \gg n$,

$$
\bar{W}(n, \tau) \approx n^{\alpha} .
$$

For both the $\mathrm{BD}$ and SD models there is a plethora of experimental and theoretical results validating the phenomenological expressions (1.6) - (1.9). For an overview of the huge literature, see [1], [8].

Stochastic PDEs are central to the analytic treatment of continuum deposition models and the attendant phenomenological surface width relations (1.6) - (1.9). The classic linear differential equation of Edwards and Wilkinson [3], which treats the SR model, provides evidence for $\alpha=1 / 2$ and $\beta=1 / 4$ in dimension $d=1$. The landmark paper [6] (KPZ) treats the BD model and accounts for lateral growth by adding non-linear terms. Subject to the validity of the KPZ methods, which are justified by plausibility arguments and which cannot be formally derived (see e.g. p. 56 of [1]), one obtains in dimension $d=1$ the 'exact' values $\alpha=1 / 2$ and $\beta=1 / 3$. Higher dimensional values of $\alpha$ and $\beta$ are not known.

Despite the abundance of experimental results and despite the successes of PDEs, there is no formal, mathematically rigorous treatment of the stochastic properties of the surface width for the BD and SR models.

In this note we address this lack of mathematical precision by providing a formal and rigorous probabilistic treatment of the mean height and surface width functionals, establishing a thermodynamic limit and Gaussian fluctuations for the mean height and surface width functionals. The mathematical underpinnings reside in the general limit theory of stabilizing functionals of marked Poisson point processes [7]. Although we do not obtain mathematical values for the roughness and growth exponents, the present approach provides additional information on $\alpha$. Attention here is confined to the continuum, but the results also hold in the lattice setting.

\section{Statement of Results}

\subsection{Thermodynamic limits}

Denote by $\mathcal{P}_{\tau}$ a rate one homogeneous Poisson point process on $\mathbb{R}^{d} \times[0, \tau]$. Without loss of generality, for all $n=1,2, \ldots$ we let $\mathcal{P}_{n, \tau}$ be the restriction of $\mathcal{P}_{\tau}$ to $Q_{n} \times[0, \tau]$.

Intuitively, one expects that the large $n$ limit of each summand in the random height functional $H(n, \tau):=\sum_{P^{\prime} \in A(n, \tau)} h\left(P_{i}^{\prime}\right)$ is related to a height functional defined on the 'infinite interface' created by the deposition of particles in $\mathcal{P}_{\tau}$ on the infinite substrate $\mathbb{R}^{d}$. However, it is not straightforward to make rigorous sense of the notion of sequential deposition of an infinite number of particles over a substrate of infinite extent. 
We adjoin to $\mathcal{P}_{\tau}$ a point $\{\boldsymbol{0}\}:=\{(0, t)\}$, with spatial coordinate the origin of $\mathbb{R}^{d}$ and time coordinate uniformly distributed over $[0, \tau]$. The collection of points $\mathcal{P}_{\tau} \cup\{\boldsymbol{0}\}$ forms a Palm distribution and we use it to define 'typical' height and squared width fluctuation functionals. Let $\mathbf{0}^{\prime} \in \mathbb{R}^{d} \times[0, \tau]$ be the random position of $\{\mathbf{0}\}$ after displacement with respect to the incoming particles $\mathcal{P}_{n, \tau}, h\left(\mathbf{0}^{\prime}\right):=$ $h\left(\mathcal{P}_{n, \tau} \cup\{\mathbf{0}\}, \mathbf{0}^{\prime}\right)$ its random height, and $A(n, \tau, 0)$ the active zone generated by $\mathcal{P}_{n, \tau} \cup\{\mathbf{0}\}$.

The following thermodynamic limits hold for both the BD and SR models. They involve notions of conditional expectation and conditional variance. Given a a random variable $\zeta$ and an event $B$ with non-zero probability, the conditional distribution of $\zeta$ given $B$ is the probability distribution $P[\{\zeta \in \cdot\} \cap B] / P[B]$; the mean and variance of this conditional distribution are denoted $E[\zeta \mid B]$ and $\operatorname{Var}[\zeta \mid B]$.

Theorem 2.1 (a) For all $\tau \in(0, \infty)$, the random limits

$$
h_{0}(\tau):=\lim _{n \rightarrow \infty} h\left(\mathbf{0}^{\prime}\right) \cdot 1_{\left\{\mathbf{0}^{\prime} \in A(n, \tau, 0)\right\}} \text { and } \quad \xi_{0}(\tau):=\lim _{n \rightarrow \infty} 1_{\left\{\mathbf{0}^{\prime} \in A(n, \tau, 0)\right\}}
$$

exist a.s.

(b) For all $\tau \in(0, \infty)$,

$$
\lim _{n \rightarrow \infty} \frac{|A(n, \tau)|}{n^{d}}=\tau \cdot E\left[\xi_{0}(\tau)\right] \text { a.s. }
$$

(c) For all $\tau \in(0, \infty)$,

$$
\lim _{n \rightarrow \infty} \bar{H}\left(\mathcal{P}_{n, \tau}\right)=\frac{E\left[h_{0}(\tau)\right]}{E\left[\xi_{0}(\tau)\right]}=E\left[h_{0}(\tau) \mid \xi_{0}(\tau)=1\right] \text { a.s. }
$$

If we interpret $h\left(\mathbf{0}^{\prime}\right) \cdot 1_{\left\{\mathbf{0}^{\prime} \in A(n, \tau, 0)\right\}}$ as the height of a 'typical' point in the interface, then the 'typical' squared width (height fluctuation) of a point in the growing interface $A(n, \tau, 0)$ is given by

$$
w\left(\mathbf{0}^{\prime}\right) \cdot 1_{\left\{\mathbf{0}^{\prime} \in A(n, \tau, 0)\right\}}:=w\left(\mathcal{P}_{n, \tau} \cup\{\mathbf{0}\}, \mathbf{0}^{\prime}\right) \cdot 1_{\left\{\mathbf{0}^{\prime} \in A(n, \tau, 0)\right\}} .
$$

The next result shows that the typical squared width a.s. converges in the large $n$ limit, and moreover the limit governs the fluctuations of the interface width.

Theorem 2.2 (a) For all $\tau \in(0, \infty)$, the limit

$$
w_{0}(\tau):=\lim _{n \rightarrow \infty} w\left(\mathbf{0}^{\prime}\right) \cdot 1_{\left\{\mathbf{0}^{\prime} \in A(n, \tau, 0)\right\}}
$$

exists a.s. 
(b) For all $\tau \in(0, \infty)$,

$$
\begin{aligned}
\lim _{n \rightarrow \infty} \bar{W}^{2}\left(\mathcal{P}_{n, \tau}\right):=\lim _{n \rightarrow \infty} \frac{1}{|A(n, \tau)|} \sum_{P_{i}^{\prime} \in A(n, \tau)} w\left(P_{i}^{\prime}\right)= & \frac{E\left[h_{0}^{2}(\tau)\right]}{E\left[\xi_{0}(\tau)\right]}-\left(\frac{E\left[h_{0}(\tau)\right]}{E\left[\xi_{0}(\tau)\right]}\right)^{2} \\
& =\operatorname{Var}\left[h_{0}(\tau) \mid \xi_{0}(\tau)=1\right] \\
& =E\left[w_{0}(\tau) \mid \xi_{0}(\tau)=1\right] \text { a.s. }
\end{aligned}
$$

\section{Remarks}

(i) Just as the random variable $h_{0}(\tau)$ gives meaning to the height of a 'typical' particle in an infinite interface defined by a Poisson point process, $w_{0}(\tau)$ may be interpreted as a 'typical' width. Similarly, $E\left[\xi_{0}(\tau)\right]$ is the probability that a typical particle belongs to the active zone.

(ii) The scientific literature assumes the existence of the $\operatorname{limits}_{\lim } \rightarrow \infty \bar{H}(n, \tau)$ and $\lim _{n \rightarrow \infty} \bar{W}^{2}(n, \tau)$. Theorems 2.1 and 2.2 puts this on solid footing and identifies the limiting constants. In statistical terms, Theorems 2.1 (b) and 2.2 (c) say that the sample mean (variance) of the heights of the particles in the interface form a consistent estimator for the mean (variance) of the random height of a typical particle, conditional on its being in the interface at all, as the substrate becomes large.

(iii) For both the $\mathrm{BD}$ and $\mathrm{SR}$ models, it is of great interest to estimate the constants in Theorems 2.1(c) and 2.2(b). Heuristically, one expects that $E\left[h_{0}(\tau) \mid \xi_{0}(\tau)=\right.$ $1] \approx C \cdot \tau$ since the expected height should vary directly with the deposition intensity $\tau$. For large $n$, we expect that $\mathbf{0}^{\prime} \in A(n, \tau, 0)$ with a probability inversely proportional to $\tau$, i.e., we expect $E\left[\xi_{0}(\tau)\right] \approx 1 / \tau$. Also, given $\xi_{0}(\tau)=1$, one expects that the expectation of the squared width of a 'typical' particle in the smoothed interface, namely $E\left[w_{0}(\tau) \mid \xi_{0}(\tau)=1\right]$ is upper bounded by the expectation of the width of a 'typical' particle in the non-smoothed interface. The latter is just the variance of a Poisson random variable with parameter $\tau$, giving $E\left[w_{0}(\tau) \mid \xi_{0}(\tau)=1\right]=O(\tau)$, which is consistent with the simulations of Zabolitzky and Stauffer ([9], p. 1529). Whether this bound can be improved is the subject of widespread research. A rigorous mathematical treatment of $E\left[w_{0}(\tau) \mid \xi_{0}(\tau)=1\right]$ would be welcome.

\subsection{Gaussian fluctuations}

Both the height functional $H$ and a modified version of surface width exhibit Gaussian fluctuations about the mean, as shown by the following distributional results. $N\left(0, \sigma^{2}\right)$ denotes a normal random variable with mean 0 and variance $\sigma^{2}$, and $\stackrel{\mathcal{D}}{\longrightarrow}$ denotes convergence in distribution. 
Theorem 2.3 For all $\tau \in(0, \infty)$ there exists $\sigma_{\tau}^{2}:=\sigma_{\tau}^{2}(H)>0$ such that

$$
\frac{H\left(\mathcal{P}_{n, \tau}\right)-E H\left(\mathcal{P}_{n, \tau}\right)}{n^{d / 2}} \stackrel{\mathcal{D}}{\longrightarrow} N\left(0, \sigma_{\tau}^{2}\right)
$$

and $n^{-d} \operatorname{Var} H\left(\mathcal{P}_{n, \tau}\right) \rightarrow \sigma_{\tau}^{2}$

Let $D(\tau):=\frac{E\left[h_{0}(\tau)\right]}{E\left[\xi_{0}(\tau)\right]}$ denote the mean deposition height given by Theorem 2.1(c). The following is closely related to the surface width functional

$$
W_{1}\left(\mathcal{P}_{n, \tau}\right):=W_{1}(n, \tau):=\sum_{P_{i}^{\prime} \in A(n, \tau)}\left(h\left(P_{i}^{\prime}\right)-D(\tau)\right)^{2} .
$$

The modified width functional $W_{1}$ exhibits Gaussian fluctuations around its mean in the large $n$ limit.

Theorem 2.4 For all $\tau \in(0, \infty)$ there exists $\sigma_{\tau}^{2}:=\sigma_{\tau}^{2}\left(W_{1}\right)>0$ such that

$$
\frac{W_{1}\left(\mathcal{P}_{n, \tau}\right)-E W_{1}\left(\mathcal{P}_{n, \tau}\right)}{n^{d / 2}} \stackrel{\mathcal{D}}{\longrightarrow} N\left(0, \sigma_{\tau}^{2}\right)
$$

and $n^{-d} \operatorname{Var} W_{1}\left(\mathcal{P}_{n, \tau}\right) \rightarrow \sigma_{\tau}^{2}$

Remarks

(i) If the Poisson point process $\mathcal{P}_{n, \tau}$ is replaced by the binomial point process $\left\{U_{n, 1}, \ldots, U_{n,[n \tau]}\right\}$, where $U_{n, 1}, U_{n, 2}, \ldots$ are i.i.d. with the uniform distribution on $Q_{n} \times$ $[0, \tau]$, then Theorems 2.1 and 2.2 continue to hold. Theorems 2.3 and 2.4 also remain valid, albeit with smaller variances $\sigma_{\tau}^{2}(H)$ and $\sigma_{\tau}^{2}\left(W_{1}\right)$. See [7] for details.

(ii) Theorems 2.1-2.4 hold for particles of random shapes and types; the particles need not be balls [7]. The substrate $Q_{n}$ need not be a cube, but may be a more general region subject to a smoothness assumption on its boundary [7].

(iii) We have not been able to determine the dependence of $\sigma_{\tau}^{2}(H)$ and $\sigma_{\tau}^{2}\left(W_{1}\right)$ on $\tau$. The exact order of magnitude of $\sigma_{\tau}^{2}(H)$ and $\sigma_{\tau}^{2}\left(W_{1}\right)$ could help determine the scaling exponents $\alpha$ and $\beta$ in (1.6) - (1.8).

(iv) Extending Theorems 2.1-2.4 to cases involving $\tau$ and $n$ going to infinity together appears beyond the scope of the methods here. Such cases are of interest and merit investigation, particularly since they might also help determine values for $\alpha$ and $\beta$. 


\section{Methods}

To prove limit results for the mean height and width functionals, we write

$$
\bar{H}(n, \tau):=\bar{H}\left(\mathcal{P}_{n, \tau}\right):=\frac{n^{d}}{|A(n, \tau)|} \frac{\sum_{P_{i}^{\prime} \in A(n, \tau)} h\left(P_{i}^{\prime}\right)}{n^{d}}
$$

and

$$
\bar{W}^{2}(n, \tau):=\bar{W}^{2}\left(\mathcal{P}_{n, \tau}\right):=\frac{n^{d}}{|A(n, \tau)|} \frac{\sum_{P_{i}^{\prime} \in A(n, \tau)} w\left(P_{i}^{\prime}\right)}{n^{d}} .
$$

To prove a thermodynamic limit for $\bar{H}\left(\mathcal{P}_{n, \tau}\right)$ it will suffice to show that the quotients $\frac{n^{d}}{|A(n, \tau)|}$ and $\frac{\sum_{P_{i}^{\prime} \in A(n, \tau)} h\left(P_{i}^{\prime}\right)}{n^{d}}$ both tend to a limit a.s. The same is true for $\bar{W}\left(\mathcal{P}_{n, \tau}\right)$. Notice that we may write

$$
|A(n, \tau)|:=\sum_{P_{i} \in \mathcal{P}_{n, \tau}} \xi\left(\mathcal{P}_{n, \tau}, P_{i}\right)
$$

where $\xi(\mathcal{X}, x)=1$ or 0 , depending upon whether $x$ is exposed or not in the agglomeration induced by $\mathcal{X}$.

To extract limits for the height and width functionals, as well as for the number of exposed points, we probe their behavior by adjoining $\{\mathbf{0}\}$ to $\mathcal{X}$, where we recall that $\{\mathbf{0}\}$ has as spatial coordinate the origin of $\mathbb{R}^{d}$ and a time coordinate randomly distributed over $[0, \tau]$. The change in the height functional (the 'add one cost' [7]) caused by the insertion of $\{\mathbf{0}\}$ is given by

$$
\Delta_{H}(\mathcal{X}):=H(\mathcal{X} \cup\{\mathbf{0}\})-H(\mathcal{X})
$$

with a similar definition for $\Delta_{W}(\mathcal{X})$ and $\Delta_{W_{1}}(\mathcal{X})$.

The displacement of the incoming particle $\{\mathbf{0}\}$ is clearly determined by the location and displacement of particles previously arriving within a distance $D_{3}:=$ $2\left(D_{1}+D_{2}\right)$ of the origin of $\mathbb{R}^{d}$. Whether the particle ends up in the active zone $A(\mathcal{X})$ is clearly a function of particles arriving within a distance $D_{3}$ of the origin.

Also, the insertion of the particle $\{\mathbf{0}\}$ will only affect a later arriving particle at location $x \in \mathbb{R}^{d}$ if there is a sequence of points $\left\{\left(x_{i}, t_{i}\right)\right\}_{i=1}^{n}$ in $\mathcal{X}$ such that

$$
\begin{gathered}
\left|x_{1}\right| \leq D_{3}, \\
\left|x_{i}-x_{i+1}\right| \leq D_{3}, \quad \text { and } t_{i}<t_{i+1}, \quad 1 \leq i \leq n-1,
\end{gathered}
$$

and

$$
\left|x_{n}-x\right| \leq D_{3}
$$


When $\mathcal{X}$ is the homogeneous Poisson point process $\mathcal{P}_{n, \tau}$, then such sequences exist with a probability decaying exponentially in $|x|[7]$. Hence, adjoining $\{\mathbf{0}\}$ to $\mathcal{P}_{n, \tau}$ changes the heights $h\left(P_{i}^{\prime}\right)$ of the re-positioned particles $P_{i}^{\prime}, P_{i} \in \mathcal{P}_{n, \tau},\left|P_{i}\right| \geq$ $\lambda$, with a probability which decays exponentially in $\lambda$. (Thus $h\left(P_{i}^{\prime}\right)$ and $\xi\left(P_{i}^{\prime}\right)$ have exponentially decaying correlations.) The insertion of $\{\mathbf{0}\}$ thus changes the morphology of the active zone at distances larger than $\lambda$ from the origin with a probability decaying exponentially in $\lambda$.

We conclude that for all deposition intensities $\tau \in(0, \infty)$, there is an a.s. finite random variable $N:=N_{H}(\tau)$, with exponentially decaying tails, and an a.s. finite random variable $\Delta(\tau):=\Delta_{H}(\tau)$ such that with probability one

$$
H\left(\left(\mathcal{P}_{\tau} \cap Q_{N}\right) \cup\{\boldsymbol{0}\} \cup \mathcal{A}\right)-H\left(\left(\mathcal{P}_{\tau} \cap Q_{N}\right) \cup \mathcal{A}\right)=\Delta(\tau)
$$

for all finite $\left.\mathcal{A} \subset\left(\mathbb{R}^{d} \backslash Q_{N}\right) \times[0, \tau]\right)$; see [7] for details. The 'add one cost' to the height functional is unaffected by changes in the particle configuration outside $Q_{N} \times[0, \tau]$. Following extant terminology, [7], we say that the height functional 'stabilizes'.

Moreover, for the same reasons, $h$ also 'stabilizes' in the sense that there are a.s. finite random variables $N$ and $h_{0}(\tau)$ such that

$$
h\left(\left(\mathcal{P}_{\tau} \cap Q_{N}\right) \cup\{\mathbf{0}\} \cup \mathcal{A}, \mathbf{0}^{\prime}\right) \cdot 1_{\left\{\mathbf{0}^{\prime} \in A\left(\left(\mathcal{P}_{\tau} \cap Q_{N}\right) \cup\{\mathbf{0}\} \cup \mathcal{A}\right)\right\}}=h_{0}(\tau)
$$

for all finite $\mathcal{A} \subset\left(\mathbb{R}^{d} \backslash Q_{N}\right) \times[0, \tau]$. This last fact yields Theorem 2.1(a).

Next, given a subcube $Q \subset \mathbb{R}^{d}$, let $U_{1, Q}, U_{2, Q}, \ldots$ be i.i.d. uniformly distributed random variables on $Q \times[0, \tau]$ and let $\mathcal{U}_{m, Q}:=\left\{U_{1, Q}, U_{2, Q}, \ldots, U_{m, Q}\right\}$ be a binomial point process. Adjoin the particle $\{\mathbf{0}\}$ to $\mathcal{U}_{m, Q}$ and consider the add one cost

$$
\Delta_{H}\left(\mathcal{U}_{m, Q}\right):=H\left(\mathcal{U}_{m, Q} \cup\{\mathbf{0}\}\right)-H\left(\mathcal{U}_{m, Q}\right) .
$$

Since $H$ stabilizes and since $N_{H}(\tau)$ has finite moments of all orders [7], by coupling binomial and Poisson point processes we can show that $\Delta_{H}\left(\mathcal{U}_{m, Q}\right)$ has finite moments of all orders. In fact, for all $\tau \in[0, \infty)$ and all $p>0, H$ satisfies the bounded moments condition

$$
\sup _{Q} \sup _{m \in[\tau|Q| / 2,3 \tau|Q| / 2]} E\left[\Delta_{H}\left(\mathcal{U}_{m, Q}\right)^{p}\right]<\infty
$$

where the first supremum runs over cubes $Q \subset \mathbb{R}^{d}$ containing the origin [7].

Likewise, the functional $h$ satisfies the bounded second moments condition

$$
\sup _{Q} \sup _{m \in[\tau|Q| / 2,3 \tau|Q| / 2]} E\left[h\left(\mathcal{U}_{m, Q} \cup\{\mathbf{0}\}, \mathbf{0}^{\prime}\right)^{2}\right]<\infty
$$


By combining the translation invariance of $h$, the uniform bound $H(\mathcal{X}) \leq$ $C_{1}(\operatorname{card}(\mathcal{X}))^{2}$, the bounded moments conditions $(3.8,3.9)$, and by invoking the stabilization of $h$ (3.6), one verifies the hypotheses of the general law of large numbers given by Theorem 3.2 of [7]. We thus deduce for all $\tau \in(0, \infty)$,

$$
\lim _{n \rightarrow \infty} \frac{1}{n^{d}} \sum_{P_{i}^{\prime} \in A(n, \tau)} h\left(P_{i}^{\prime}\right)=\tau \cdot E\left[h_{0}(\tau)\right] \quad \text { a.s. }
$$

Similarly, using the stabilization of $\xi$ and again using the general law of large numbers of [7], we may show for all $\tau \in(0, \infty)$ that $\lim _{n \rightarrow \infty} \frac{|A(n, \tau)|}{n^{d}}=\tau \cdot E\left[\xi_{0}(\tau)\right]$. This gives Theorem 2.1 (b). Combining this last limit with (3.10) and (3.1), we obtain Theorem 2.1(c).

By invoking the stabilization of $H$, we may apply the general central limit theorem (Theorem 3.1) of [7] to deduce Theorem 2.3. According to Theorem 3.1 of [7], the limiting variance $\sigma_{\tau}^{2}(H)$ is non-zero provided that $\Delta_{H}(\tau)$ is non-degenerate. To check non-degeneracy of $\Delta_{H}(\tau)$, observe that there is positive probability that the set $\mathcal{P}_{n, \tau}$ puts no particles within distance $D_{3}$ of the origin, so that in this case the difference

$$
H\left(\left(\mathcal{P}_{\tau} \cap Q_{N}\right) \cup\{\mathbf{0}\} \cup \mathcal{A}\right)-H\left(\left(\mathcal{P}_{\tau} \cap Q_{N}\right) \cup \mathcal{A}\right)
$$

is just the height of a single particle resting on the substrate. On the other hand, there is also positive probability that the set $\mathcal{P}_{n, \tau}$ is such that the addition of the origin results in a net increase of one exposed point to the active surface, in such a way that the height is increased by the height of a particle which is several layers removed from the substrate. This produces a different value for (3.11), showing the non-degeneracy of $\Delta_{H}(\tau)$.

We prove Theorems 2.2 and 2.4 in a similar fashion. Define the functionals

$$
w_{1}\left(X^{\prime}\right):=w_{1}\left(\mathcal{X}, X^{\prime}\right):=\left(h\left(X^{\prime}\right)-D(\tau)\right)^{2}
$$

and

$$
W_{1}(\mathcal{X}):=\sum_{X_{i}^{\prime} \in A(\mathcal{X})} w_{1}\left(\mathcal{X}, X_{i}^{\prime}\right) .
$$

Analogously to (3.6), the functional

$$
w_{1}\left(\mathcal{P}_{n, \tau} \cup\{\mathbf{0}\}, \mathbf{0}^{\prime}\right):=\left(h\left(\mathbf{0}^{\prime}\right)-D(\tau)\right)^{2}
$$

stabilizes in the sense that for all $\tau \in(0, \infty)$ there is a random variable $N$ with exponentially decaying tails and $w_{0}(\tau)$ a.s. finite such that

$$
w_{1}\left(\left(\mathcal{P}_{\tau} \cap Q_{N}\right) \cup\{\mathbf{0}\} \cup \mathcal{A}, \mathbf{0}^{\prime}\right) \cdot 1_{\left\{\mathbf{0}^{\prime} \in A(n, \tau, 0)\right\}}=w_{0}(\tau)
$$


for all finite $\mathcal{A} \subset\left(\mathbb{R}^{d} \backslash Q_{N}\right) \times[0, \tau]$.

Moreover, since $W_{1}$ satisfies the bounded moments condition (3.8) (replace $\Delta_{H}$ by $\Delta_{W_{1}}$ there) and since $w_{1}$ satisfies the bounded second moments condition (3.9) (replace $h$ by $w_{1}$ there), we obtain for all $\tau \in(0, \infty)$,

$$
w_{0}(\tau)=\lim _{n \rightarrow \infty} w_{1}\left(\mathcal{P}_{n, \tau} \cup\{\mathbf{0}\}, \mathbf{0}^{\prime}\right) \cdot 1_{\left\{\mathbf{0}^{\prime} \in A(n, \tau, 0)\right\}} \text { a.s. }
$$

Since $\bar{H} \rightarrow D(\tau)$ a.s. by Theorem 2.1(c), it follows that

$$
w_{0}(\tau)=\lim _{n \rightarrow \infty} w\left(\mathcal{P}_{n, \tau} \cup\{\mathbf{0}\}, \mathbf{0}^{\prime}\right) \cdot 1_{\left\{\mathbf{0}^{\prime} \in A(n, \tau, 0)\right\}} \text { a.s. }
$$

i.e., Theorem 2.2(a) holds.

To prove Theorem $2.2(\mathrm{~b})$, we write

$$
W\left(\mathcal{P}_{n, \tau}\right)=\sum_{P_{i}^{\prime} \in A(n, \tau)}\left(h\left(P_{i}^{\prime}\right)-\bar{H}(n, \tau)\right)^{2}=\sum_{P_{i}^{\prime} \in A(n, \tau)} h^{2}\left(P_{i}^{\prime}\right)-|A(n, \tau)|(\bar{H}(n, \tau))^{2} .
$$

Dividing both sides of (3.13) by $A(n, \tau)$ and appealing to Theorem 2.1(c), we see that to establish a thermodynamic limit for $\bar{W}\left(\mathcal{P}_{n, \tau}\right)$ it is enough to show a thermodynamic limit for $\frac{\sum_{P_{i}^{\prime} \in A(n, \tau)} h^{2}\left(P_{i}^{\prime}\right)}{|A(n, \tau)|}$. However, by following the proof of Theorem 2.1 with $h$ replaced by $h^{2}$, we find that

$$
\lim _{n \rightarrow \infty} \frac{\sum_{P_{i}^{\prime} \in A(n, \tau)} h^{2}\left(P_{i}^{\prime}\right)}{|A(n, \tau)|}=\frac{E\left[h_{0}^{2}(\tau)\right]}{E\left[\xi_{0}(\tau)\right]} .
$$

Using Theorem 2.1(c) on the second term in (3.13), we obtain the first equality in Theorem 2.2(b).

The second equality in Theorem 2.2(b) is just the definition of conditional variance and it remains to show the last equality. Let $E_{n}:=\left\{\mathbf{0}^{\prime} \in A(n, \tau, 0)\right\}$ for simplicity. We have

$$
\begin{gathered}
\left(h_{0}(\tau)-D(\tau)\right)^{2} \\
=\left(\lim _{n \rightarrow \infty} h\left(\mathbf{0}^{\prime}\right) 1_{E_{n}}-D(\tau)\right)^{2} \\
=\lim _{n \rightarrow \infty} h^{2}\left(\mathbf{0}^{\prime}\right) \cdot 1_{E_{n}}-2 D(\tau) \lim _{n \rightarrow \infty} h\left(\mathbf{0}^{\prime}\right) 1_{E_{n}}+D(\tau)^{2} \\
=\lim _{n \rightarrow \infty}\left(h\left(\mathbf{0}^{\prime}\right)-D(\tau)\right)^{2} \cdot 1_{E_{n}}+D(\tau)^{2} \lim _{n \rightarrow \infty} 1_{E_{n}^{c}} \\
=\lim _{n \rightarrow \infty} w_{1}\left(\mathcal{P}_{n, \tau} \cup\{\mathbf{0}\}, \mathbf{0}^{\prime}\right) \cdot 1_{E_{n}}+D(\tau)^{2} \lim _{n \rightarrow \infty} 1_{E_{n}^{c}} \\
=w_{0}(\tau)+D(\tau)^{2}\left(1-\xi_{0}(\tau)\right),
\end{gathered}
$$

by (3.12) and by Theorem 2.1(a). Thus, by the above identity

$$
\begin{array}{r}
\operatorname{Var}\left[h_{0}(\tau) \mid \xi_{0}(\tau)=1\right]=E\left[\left(h_{0}(\tau)-E\left[h_{0}(\tau) \mid \xi_{0}(\tau)=1\right]\right)^{2} \mid \xi_{0}(\tau)=1\right] \\
=E\left[\left(h_{0}(\tau)-D(\tau)\right)^{2} \mid \xi_{0}(\tau)=1\right]=E\left[w_{0}(\tau) \mid \xi_{0}(\tau)=1\right]
\end{array}
$$


showing the last equality in Theorem 2.2(b).

Finally, we address the proof of Theorem 2.4. Exactly as for the height functional $H$, we can show that $W_{1}$ stabilizes in the sense that (3.5) holds with $H$ replaced by $W_{1}$. Invoking the stabilization of $W_{1}$ and applying Theorem 3.1 of [7], we obtain Theorem 2.4. Non-degeneracy of $\Delta_{W_{1}}(\tau)$ follows as in the proof of non-degeneracy of $\Delta_{H}(\tau)$.

\section{References}

[1] A.-L. Barabási and H. E. Stanley (1995) Fractal Concepts in Surface Growth, Cambridge University Press.

[2] D. J. Cumberland and R.J. Crawford (1987) The Packing of Particles, in the Handbook of Powder Technology, 6, Elsevier.

[3] S. F. Edwards and D. R. Wilkinson (1982) The surface statistics of a granular aggregate, Proc. R. Soc. London A 381, 17-31.

[4] F. Family (1986) Scaling of rough surfaces: effects of surface diffusion, J. Phys. A 19, L441-L446.

[5] F. Family and T. Vicsek (1985) Scaling of the active zone in the Eden process on percolation networks and the ballistic deposition model, J. Phys. A 18, L75-L81.

[6] M. Kardar, G. Parisi, and Y. Zhang (1986) Dynamic scaling of growing interfaces, Phys. Rev. Letters 56, 9, 889-892.

[7] M. D. Penrose and J. E. Yukich (2000) Limit theory for random sequential packing and deposition, preprint.

[8] T. Vicsek (1989) Fractal Growth Phenomena, World Scientific, Singapore.

[9] J. G. Zabolitzky and D. Stauffer (1986) Simulation of large Eden clusters, Phys. Rev. A, 34, 2, 1523-1530. 